\title{
Analysis of the academic production of the Faculty of Sociology of the Santo Tomás University, from the fields of action of the same university
}

\author{
Miguel Urra Canales \\ Decano de la Facultad de Sociología \\ Grupo de Investigación "Estudios Interdisciplinarios de la Sociedad y la Cultura"
}

\begin{abstract}
:
This article aims to analyze the alignment of research products of the Faculty of Sociology of the Santo Tomás University with these fields of action and institutional interactions. For this, a bibliometric exercise is carried out with 104 research products of the faculty's teachers. As main results, a focus is obtained in the areas of "Peace and coexistence", "Social groups" and "Citizen participation". As the main conclusion, there is an adequate alignment of research products with the "Society" field of action and a still shy alignment with the "Environment" field of action.
\end{abstract}

Keywords: fields of action, academic production, society, environment 


\section{1.- Approach}

Between 2012 and 2018, the Santo Tomás University developed an information systematization work, intending to define its national stakes and identify its fields of action, taking into account its institutional capacities and strengths in teaching, research and social projection of the different academic programs in Headquarters and Sectionals. This work was led by the General Academic Vice Rectory, together with the Units of Curriculum Development and Teacher Training, Research and University Social Responsibility. (Ostos, O.L., and Cortés, M.A. 2018)

In the institutional documents of the USTA, such as the 2012-2015 Development Plan or the 20162027 Multicampus Integral Plan, it is firmly committed to concentrate efforts and focus projects in some specific areas, to multiply the impacts. As a consequence of this, the fields of action were defined as "areas or spaces of significant reality, with relative limits, where the substantive functions converge from rigorous investigative processes, about the problems that have been identified and that are of interest for the USTA, with evidence of context, experience, and interdisciplinarity "(Cedeño, 2016).

Finally, after a long process of analysis and deliberation, two main fields of action were defined: society and the environment. Each of them includes a series of interactions, that is, areas where the USTA has shown greater intellectual and academic production. They are the following (Ostos, O.L., and Cortés, M.A. 2018: 36):

\section{SOCIETY}

1. Communication

2. Economic and social development

3. Public Policy

4. Human rights

5. Economy

6. Information literacy

7. Knowledge management

8. Social behavior

9. Social systems

10. Health, Welfare and public health

11. Social groups

12. Value systems

13. Education

14. Infrastructure

15. Urban planning 
16. Information users

17. Peace and coexistence

18. Citizen Participation

19. Scientific developments applied to the transformation of society

20. Equality and justice

ENVIRONMENT

1. Environments

2. Biodiversity

3. Habitat

4. Ecology

5. Atmosphere

6. Aquatic ecosystems

7. Terrestrial ecosystems

8. Effects of human activities

9. Environmental management

10. Pollution

11. Clean and sustainable energies

12. Sustainable cities

13. Smart cities

14. Responsible production and consumption

With these definitions, this article aims to analyze the alignment of the research products of the Faculty of Sociology of the Santo Tomás University with these fields of action and institutional interactions. This work will help to evaluate the strengths and weaknesses of the Faculty and to project research, teaching and social projection strategies for the future. It should be noted that at no time is this article done to evaluate the individual production or the trajectory of each teacher. 


\section{2.- Methodology}

In the first place, 104 research products of the faculty teachers have been identified (69\% articles, $13 \%$ books, $6 \%$ doctoral thesis, $5 \%$ book chapters, $5 \%$ master thesis, and $2 \%$ papers). With these products, a table was constructed that crossed them with the 34 interactions of the 2 fields of action. Each product and each interaction was given a value between 0 and 10, according to their degree of affinity ( 0 means that it has no relationship with the interaction and 10 that the relationship with the interaction is total). In the end, a table of 3,675 cells was achieved, which consolidates a systematization clear enough to yield the results presented below.

\section{3.-Results}

\section{1.-Society}

As already indicated in the approach, the field of action "society" has 20 interactions. Of these, the research production of teachers works in 18, leaving out "Health, welfare and public health" and "Literacy." This is interesting since sociology works all fields where social interactions occur and, precisely, two fields that belong (if this expression can be used in the academic world) to the faculties of Education and Science of the Health of the different headquarters and sections of the USTA.

Below is the score obtained in each of the fields.

Table 1: Interactions of the "Society" field of action

\begin{tabular}{|l|c|}
\hline Peace and coexistence & 184 \\
\hline Social groups & 144 \\
\hline Citizen participation & 124 \\
\hline Social behavior & 95 \\
\hline Town planning & 93 \\
\hline Public politics & 90 \\
\hline Equality and justice & 74 \\
\hline Social Systems & 70 \\
\hline Human rights & 69 \\
\hline Economic and Social & \\
Development & 66 \\
\hline Economy & 57 \\
\hline Education & 53 \\
\hline Values system & 47 \\
\hline Infrastructure & 20 \\
\hline Scientific developments & 19 \\
\hline Knowledge management & 12 \\
\hline Communication & 12 \\
\hline Information Users & 5 \\
\hline
\end{tabular}




\begin{tabular}{|l|l|}
\hline $\begin{array}{l}\text { Health, welfare and public } \\
\text { health }\end{array}$ & 0 \\
\hline Literacy & 0 \\
\hline
\end{tabular}

With this table, we can do three great analyzes. The first, on the groups of topics worked; the second, on the relative weight of each topic and the third, the crossing of these topics with the curriculum.

As for the first, there are 3 large groups:

- The ones that work the most (they exceed 100 points), which we can call structural.
○ Peace and coexistence
- Social groups
- Citizen participation

- Those who work in a temporary manner (between 50 and 99 points).
- Social Behavior
- Urbanism
- Public Policy
- Equality and justice
- Social Systems
○ Human Rights
- Economic and Social Development
- Economy
- Education

- Those who work sporadically (less than 50 points).
○ Value system
- Infrastructure
- Scientific developments
- Knowledge management
- Communication
- Information users

Regarding the relative weight of each topic, it can be calculated that, with 104 articles, an interaction that appeared strongly (10 points) in all of them, would add a total of 1040 points. On this maximum score, not with an appreciative spirit, but with the intention of establishing a baseline on which to continue measuring, the weight of each topic would be as follows:

Table 2: Relative weight of the interactions of the "Society" field of action

\begin{tabular}{|l|c|}
\hline Peace and coexistence & $18 \%$ \\
\hline Social groups & $14 \%$ \\
\hline Citizen participation & $12 \%$ \\
\hline Social behavior & $9 \%$ \\
\hline Town planning & $9 \%$ \\
\hline Public politics & $9 \%$ \\
\hline
\end{tabular}




\begin{tabular}{|l|c|}
\hline Equality and justice & $7 \%$ \\
\hline Social Systems & $7 \%$ \\
\hline Human rights & $7 \%$ \\
\hline Economic and Social Development & $6 \%$ \\
\hline Economy & $5 \%$ \\
\hline Education & $5 \%$ \\
\hline Values system & $5 \%$ \\
\hline Infrastructure & $2 \%$ \\
\hline Scientific developments & $2 \%$ \\
\hline Knowledge management & $1 \%$ \\
\hline Communication & $1 \%$ \\
\hline Information Users & $0 \%$ \\
\hline Health, welfare and public health & $0 \%$ \\
\hline Literacy & $0 \%$ \\
\hline
\end{tabular}

In this way, it could be read, for example, that $18 \%$ of the research production of teachers of the Faculty of Sociology of the USTA, focuses on the theme of "Peace and coexistence" or that $44 \%$ of it It focuses on the areas of "Peace and Coexistence", "Social Groups" and "Citizen Participation". It is not the objective of the article but perhaps it could be affirmed that, according to the teaching staff and their research trajectories, these would be the three emphases of the program.

Finally, the majority interactions in the curriculum are strongly identified:

\begin{tabular}{|l|l|}
\hline \multicolumn{1}{|c|}{ Interacción } & \multicolumn{1}{|c|}{ Ejemplo de aparición en el plan de estudios } \\
\hline Peace and coexistence & $\begin{array}{l}\text { Seminars, research seedbeds and academic events of } \\
\text { Sociology of Violence and Sociology of Peace. }\end{array}$ \\
\hline Social groups & $\begin{array}{l}\text { Seminars, research seedbeds and academic events on } \\
\text { migration, ethnic communities or Afro studies. }\end{array}$ \\
\hline Citizen participation & $\begin{array}{l}\text { Seminars of social movements and participatory planning } \\
\text { approach in the Master of Planning for Development } \\
\text { promoted by the Faculty. }\end{array}$ \\
\hline Social behavior & It works transversely. \\
\hline Town planning & $\begin{array}{l}\text { Approach from the Master of Development Planning } \\
\text { promoted by the Faculty. }\end{array}$ \\
\hline Public politics & $\begin{array}{l}\text { Elective seminar and approach from the Master of Planning } \\
\text { for Development promoted by the Faculty. }\end{array}$ \\
\hline Equality and justice & It works transversely. \\
\hline Social Systems & It works transversely. \\
\hline Human rights & It works transversely. \\
\hline Economic and Social Development & $\begin{array}{l}\text { Academic spaces of "Sociology of Development", "Planning } \\
\text { and Management" and "Local and Regional Planning", plus } \\
\text { the approach from the Master of Planning for Development } \\
\text { promoted by the Faculty. }\end{array}$ \\
\hline
\end{tabular}




\begin{tabular}{|l|l|}
\hline Economy & Two academic spaces in the undergraduate. \\
\hline Education & $\begin{array}{l}\text { Seminars, research seedbeds and academic events of } \\
\text { Sociology of Education. }\end{array}$ \\
\hline Values system & It works transversely. \\
\hline Infrastructure & $\begin{array}{l}\text { Approach from the Master of Development Planning } \\
\text { promoted by the Faculty. }\end{array}$ \\
\hline Scientific developments & It does not appear in the curriculum. \\
\hline Knowledge management & It does not appear in the curriculum. \\
\hline Communication & It does not appear in the curriculum. \\
\hline Information Users & It does not appear in the curriculum. \\
\hline Health, welfare and public health & It does not appear in the curriculum. \\
\hline Literacy & It does not appear in the curriculum. \\
\hline
\end{tabular}

\section{2.-Enviroment}

Environmental sciences have strongly approached social sciences but, social sciences, are still shy in their academic approach to environmental sciences. This is evidenced in the production of the teachers analyzed, as shown in the following table.

Table 3: Interactions of the "Environment" field of action

\begin{tabular}{|l|c|}
\hline Habitat & 117 \\
\hline Sustainable cities & 68 \\
\hline Effects of human activities & 30 \\
\hline $\begin{array}{l}\text { Responsible production and } \\
\text { consumption }\end{array}$ & 23 \\
\hline Biodiversity & 12 \\
\hline Ecology & 12 \\
\hline Smart cities & 12 \\
\hline Surroundings & 8 \\
\hline Atmosphere & 0 \\
\hline Aquatic ecosystems & 0 \\
\hline Terrestrial ecosystems & 0 \\
\hline Environmental management & 0 \\
\hline Pollution & 0 \\
\hline Clean energies & 0 \\
\hline
\end{tabular}

These subjects are not yet worked in depth and specifically in the Faculty and have a very limited weight in the curriculum (beyond electives offered in other careers, activities developed by the research seedbeds or the particular environmental activism of teachers and students). 
3.4.-Productos más relevantes en cada campo.

Table 4: Most relevant products in the "Society" field

\begin{tabular}{|c|c|}
\hline Peace and coexistence & $\begin{array}{c}\text { (Idrobo, J. y Amaya, J. 2018) } \\
\text { (Acosta, C. et. al. 2019) } \\
\text { (Gamba, A. 2006) } \\
\text { (Aliaga, F., Uribe, Cr., Blanco, J.E., Ballén, D.A. y Robayo, I.A. } \\
\text { 2017) } \\
\text { (Narváez, G. 2016) } \\
\text { (Narváez, G. 2017) } \\
\text { (Castiblanco, C.A y Narváez, G.E. eds. 2018) }\end{array}$ \\
\hline Social groups & $\begin{array}{c}\text { (Aliaga, F. ed 2018) } \\
\text { (Gamba, A. 2017) } \\
\text { (Acosta, C. et. al. 2019) } \\
\text { (Gamba, A. 2011) } \\
\text { (Aliaga, F. 2008) } \\
\text { (Aliaga, F. 2012) } \\
\text { (Sáenz, H. 2018) } \\
\text { Lizarazo Zuluaga Sthefanía (2011) } \\
\text { (Motoa, J. y F.-X. Tinel. 2009) }\end{array}$ \\
\hline Citizen participation & $\begin{array}{l}\text { (Gamba, A. 2017) } \\
\text { (Gamba, A. 2011) } \\
\text { (Gamba, A. 2013) } \\
\text { (Urra, M. 2018) } \\
\text { (Sáenz,H. 2010) } \\
\text { (Torres Carrillo, A. y Torres Ruiz, A.C. 2015) } \\
\text { (Aliaga, F. y Carretero, E. 2016) }\end{array}$ \\
\hline Social behavior & $\begin{array}{c}\text { (Varón, D.C 2014) } \\
\text { (Urra Canales, M., Acosta Oidor, C., Salazar Baena, V., Jaime } \\
\text { Ruiz, E. 2018) } \\
\text { (Urra, M. 2017) } \\
\text { (de la Rosa, L. 2013) } \\
\text { (Gamba, A. 2011) } \\
\text { (Aliga, F., Baracaldo, V., Pinto, L. y Gissi, N. 2018) }\end{array}$ \\
\hline Town planning & $\begin{array}{l}\text { (Aliaga, F. ed (2019) } \\
\text { (Sáenz,H. 2010) } \\
\text { (Pinzón, J.A. 2012a) } \\
\text { (Pinzón, J.A. 2012b) }\end{array}$ \\
\hline Public politics & $\begin{array}{c}\text { (Calderón, D. y Jaime, E. 2018) } \\
\text { (Narváez, G. 2017) } \\
\text { (Narváez, G. 2016) } \\
\text { (Aliaga-Saez, F.A. et.al. 2019) }\end{array}$ \\
\hline Equality and justice & $\begin{array}{c}\text { (Jaime, E. 2013) } \\
\text { (Acosta, C. et. al. 2019) } \\
\text { (Aliaga, F. ed. 2019) } \\
\text { (Idrobo, J. y Amaya, J. 2018) }\end{array}$ \\
\hline
\end{tabular}




\begin{tabular}{|c|c|}
\hline Social Systems & $\begin{array}{c}\text { (Gamba, A. 2011) } \\
\text { (Acosta, C. et. al. 2019) } \\
\text { (Gamba, A. 2013) } \\
\text { (Narváez, G. 2012) }\end{array}$ \\
\hline Human rights & $\begin{array}{c}\text { (Idrobo, J. y Amaya, J. 2018) } \\
\text { (Aliaga, F., Uribe, Cr., Blanco, J.E., Ballén, D.A. y Robayo, I.A. } \\
\text { 2017) } \\
\text { (Aliga, F., Baracaldo, V., Pinto, L. y Gissi, N. 2018) } \\
\text { (Aliaga, F. (ed) 2018) }\end{array}$ \\
\hline $\begin{array}{l}\text { Economic and Social } \\
\text { Development }\end{array}$ & $\begin{array}{l}\text { (Gamba, A. 2013) } \\
\text { (Urra, M. 2018) } \\
\text { (Tinel, F.X. 2015) } \\
\text { (Urra, M. 2018) } \\
\text { (Tinel, F.X. 2008) }\end{array}$ \\
\hline Economy & $\begin{array}{c}\text { (Sáenz,H. 2018) } \\
\text { (Urra, M. 2018) } \\
\text { (Urra, M. 2010) } \\
\text { (J Jaramillo, D Restrepo 2017) } \\
\text { (Acosta, C. 2019) }\end{array}$ \\
\hline Education & $\begin{array}{l}\text { (Urra Canales, M., Acosta Oidor, C., Salazar Baena, V., Jaime } \\
\text { Ruiz, E. 2018) }\end{array}$ \\
\hline Values system & $\begin{array}{c}\text { (Tinel, F.X. 2015) } \\
\text { (Aliga, F., Baracaldo, V., Pinto, L. y Gissi, N. 2018) }\end{array}$ \\
\hline Infrastructure & $\begin{array}{c}\text { (Torres Tovar, C.; Rincón García, J. y Vargas Moreno, J. 2009a) } \\
\text { (Torres C.A., Vargas J.E. 2009b) }\end{array}$ \\
\hline Scientific developments & $\begin{array}{l}\text { (Cáceres, I. E Idrobo, J. eds 2014) } \\
\text { (Aliaga, F. 2005) }\end{array}$ \\
\hline Knowledge management & $\begin{array}{c}\text { (Urra, M. 2018) } \\
\text { (Urra, M. et ál. 2019) }\end{array}$ \\
\hline Communication & (Aliaga, F.; Buenaño, D.A.; Carretero, E. y Cusot, G. 2016) \\
\hline Information Users & (Varón, D.C 2014) \\
\hline
\end{tabular}


Table 5: Most relevant products in the "Environment" field

\begin{tabular}{|c|c|}
\hline Habitat & 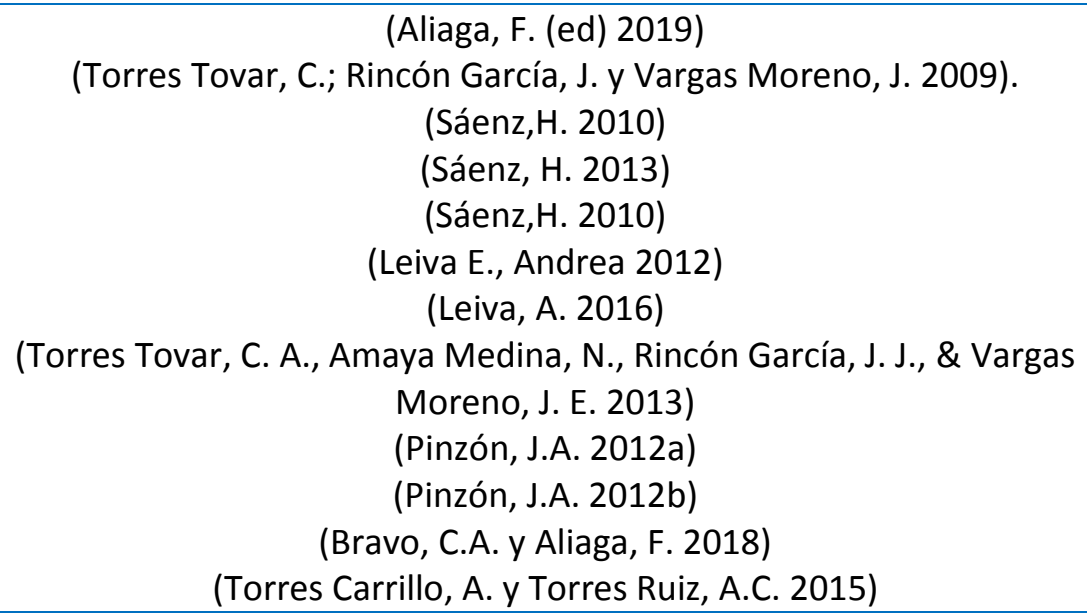 \\
\hline Sustainable cities & $\begin{array}{l}\text { Aliaga, F. (ed) 2019) } \\
\text { (Torres Tovar, C.; Rincón García, J. y Vargas Moreno, J. 2009). } \\
\text { (Sáenz,H. 2010) } \\
\text { (Sáenz, H. 2013) } \\
\text { (Sáenz,H. 2010) } \\
\text { (Torres Tovar, C. A., Amaya Medina, N., Rincón García, J. J., \& Vargas } \\
\text { Moreno, J. E. 2013) } \\
\text { (Pinzón, J.A. 2012a) } \\
\text { (Pinzón, J.A. 2012b) } \\
\text { (Torres Carrillo, A. y Torres Ruiz, A.C. 2015) }\end{array}$ \\
\hline $\begin{array}{l}\text { Effects of human } \\
\text { activities }\end{array}$ & $\begin{array}{l}\text { (Acosta, C. 2019) } \\
\text { (Tinel, F.X. 2015) }\end{array}$ \\
\hline $\begin{array}{c}\text { Responsible } \\
\text { production and } \\
\text { consumption }\end{array}$ & $\begin{array}{l}\text { (Acosta, C. 2019) } \\
\text { (Tinel, F.X. 2015) }\end{array}$ \\
\hline Biodiversity & $\begin{array}{l}\text { (Cáceres, I. E Idrobo, J. eds. 2014) } \\
\text { (Acosta, C. 2019) }\end{array}$ \\
\hline Ecology & $\begin{array}{l}\text { (Cáceres, I. E Idrobo, J. eds. 2014) } \\
\text { (Acosta, C. 2019) }\end{array}$ \\
\hline Smart cities & (Aliaga, F. ed. 2019) \\
\hline
\end{tabular}




\section{4.-Conclusions}

The main conclusion reached in this article is that there is an adequate alignment of the research products with the field of action "Society" and alignment still shy with the field of action "Environment". This, by itself, is not good or bad, nor is this bibliometry exercise designed to judge the production of teachers or the research strategy of the Faculty. The really interesting thing is that, after this analysis, there is already a baseline with which to make adjustments in the research strategy, social projection, and curriculum of the Faculty in the medium and short term.

In more detailed network analysis, it could be deepened, for example, on the authors most cited by teachers in each field and interaction. Also, this exercise could be carried out with the thesis of undergraduate students in Sociology and the Master in Development Planning. Other, more specific, lines could address, for example, gender biases in the work of some issues, funding dedicated to them or research proposals that were not developed.

As a conclusion, simply highlight the importance of systematically performing this type of exercise, to have data over time and to measure, in a certain sense, the evolution of the programs in their approach to the fields of action of the USTA.

\section{5.-References}

Acosta E, Barrantes N, Guerra-Muriel W, Maldonado-Palacios D, Silva-Lurduy J, y Aliaga, F. (2016) Apropiación y significación cultural y artística del espacio urbano: el caso del chorro de Quevedo en Bogotá. Aposta. Vol (71), pp. 62-94.

Acosta, C. (2012) Efectos del conflicto armado sobre la población civil en el Valle del Cauca durante el primer decenio del siglo XXI. Disponible en: http://bibliotecadigital.univalle.edu.co/bitstream/10893/6660/1/OBSERVADOR\%20REGIONAL\% 20No_21.pdf

Acosta, C. (2012). Anatomía del conflicto armado en el Valle del Cauca durante la primera década del siglo XXI. Guillermo de Ockham, 10(1), 83-99

Acosta, C. (2015) Transformación de la violencia paramilitar en el Valle del Cauca a partir de una presunta desmovilización. Tesis de Maestría. Disponible en: http://repositorio.flacsoandes.edu.ec/bitstream/10469/8995/2/TFLACSO-2015CAO.pdf

Acosta, C. (2019) Las huellas del desarrollo: Intersecciones entre conflicto, reconfiguración social y pacificación en Colombia. Bogotá: Ediciones USTA.

Acosta, C. et. al. (2019) Reconciliación y construcción de la paz territorial en Colombia: el caso de la comunidad nasa. Revista CIDOB d'Afers Internacionals, 121, 91-112. 
Aliaga Sáez, Felipe (2013). "Riesgos en los procesos migratorios". En Aliaga, Felipe, ed. Cultura y Migración. Enfoques Interdisciplinarios. Santiago de Compostela: Servicio de publicaciones de la Universidad de Santiago de Compostela.

Aliaga Sáez, Felipe. (2008) Algunos aspectos de los imaginarios sociales en torno al inmigrante. Aposta, revista de ciencias sociales. Universidad de Santiago de Compostela. no 39, Octubre, Noviembre y Diciembre [Consultado en febrero de 2009] [En línea] http://www.apostadigital.com/revistav3/hemeroteca/aliaga3.pdf

Aliaga, F. (2005) La construcción democrática del conocimiento tecnológico: una visión sociológica del software libre. Aposta. Vol 20, pp. 1-17.

Aliaga, F. (2006), Bolivia y Chile: Crisis, Gas, Mar e Imaginarios Sociales, Ciencias Sociales Online $3,1,1-18$.

Aliaga, F. (2008) Imaginarios Sociales, Inmigración e Integración (TIT). Tesis Doctoral

Aliaga, F. (2012) La construcción del imaginario social en torno a la integración del inmigrante desde el ámbito asociativo. Revista de Investigaciones Políticas y Sociológicas. Vol 11 (2) pp. $159-172$

Aliaga, F. (2012). El imaginario social entorno a la integración de los inmigrantes en España. TRIM, 4, 15-27. Recuperado de http://www5.uva.es/trim/TRIM/TRIM4_files/Imaginario.pdf

Aliaga, F. (ed) (2018) Migración de retorno: Colombia y otros contextos internacionales. Bogotá: Ediciones USTA.

Aliaga, F. (ed) (2019) Ciudades (in) descifrables: Imaginarios y representaciones sociales de lo urbano. Bogotá: Ediciones USTA.

Aliaga, F. (ed) (2019) Defensa de los derechos humanos de los migrantes y refugiados: el rol de las organizaciones del tercer sector en colombia y ecuador. Bogotá: Ediciones USTA.

Aliaga, F. A., Basulto, O. F. y Cabrera, J. (2012). “El grupo de discusión: elementos para la investigación en torno a los imaginarios sociales". Prisma Social, Revista de Ciencias Sociales [en línea], no 9, pp. 136-175

Aliaga, F. y Carretero, E. (2016) La comprensión del asociacionismo inmigrante en perspectiva Maffesoliana. Intersticios. Vol 10 (2).

Aliaga, F. y Carretero, E. (2016). El abordaje sociológico de los imaginarios sociales en los últimos veinte años. Espacio abierto. Cuaderno Venezolano de Sociología, 25(4), 117-128. 
Aliaga, F. y Carretero, E. (2016). El abordaje sociológico de los imaginarios sociales en los últimos veinte años. Espacio abierto. Cuaderno Venezolano de Sociología, 25(4), 117-128.

Aliaga, F. y Pintos de Cea-Naharro, J.L. (2012). La investigación en torno a los imaginarios sociales. Un horizonte abierto a las posibilidades. Revista de Investigaciones Políticas y Sociológicas, 11(2), 11-17.

Aliaga, F., Uribe, Cr., Blanco, J.E., Ballén, D.A. y Robayo, I.A. (2017) Imaginaries of the Return to Post-Conflict Colombia Discourses of Colombian Refugees in Ecuador. Dighitum. Vol 20, pp. 2437.

Aliaga, F.; Buenaño, D.A.; Carretero, E. y Cusot, G. (2016) La articulación entre comunicación política, imaginarios y emociones: un acercamiento a la Revolución Ciudadana en Ecuador. Comunicología. Vol 9 (2) pp. 167-180

Aliaga-Saez, F.A. et.al. (2019) Una innecesaria tipología para la migración de retorno. Análisis sociojurídico de la Ley para el retorno de los colombianos en el exterior. Revista Austral de Ciencias Sociales, 36, 215-232.

Aliga, F. y Escobar, G. (2006). El imaginario social del joven en Chile. Una aproximación teórica al concepto del joven problema. Aposta Digital. Revista de Ciencias Sociales. No. 31

Aliga, F., Baracaldo, V., Pinto, L. y Gissi, N. (2018) Imaginarios de exclusión y amenaza en torno al inmigrante venezolano en Colombia. Temas y Debates, 36, 61-83.

Bravo, C.A. y Aliaga, F. (2018) La producción de marginalidad urbana: El proceso socio-histórico, emergencia y configuración del Bronx en Bogotá. Imagonautas: revista Interdisciplinaria sobre imaginarios sociales, 11, 107-128.

Cáceres, I. E Idrobo, J. (eds) (2014) PARADIGMA TECNOLÓGICO Y CRISIS ECOLÓGICA: Una Reflexión Desde El Pensamiento Amerindio.

Calderón, D. y Jaime, E. (2018) La gobernanza local: una simbiosis desde y hacia el territorio. Gobernanza Multidimensional. 35-54. Bogotá: Ediciones USTA.

Castiblanco, C. (2016) La Violencia contra la ATCC en el Carare: Un campo de lucha por la definición del pasado. Tesis de maestría. Disponible en:

http://www.bdigital.unal.edu.co/54720/1/79949899.2016.pdf

Castiblanco, C. (et.al.) (2017) Protegiendo el azul, comprendí el rojo de la bandera. Bogotá: Universidad Santo Tomás.

Castiblanco, C.A y Narváez, G.E. (eds) (2018) La vida me dio otra oportunidad. Bogotá: Ediciones USTA. Disponible en: https://repository.usta.edu.co/handle/11634/11667 
Cedeño, C. L. (2016). Campos de acción. Bogotá.

Cepeda, J.,Cárdenas, J., Moreno, C. e Idrobo, J. (2016) Reflexiones en torno de la investigación universitaria. En «La Hermenéutica textual como herramienta metodológica». Bogotá: Pacheco Ed.

Chaparro, M. y Urra, M. (2013) Competencias básicas y genéricas: una visión desde los trabajadores sociales ubicados en el área de Gestión del Talento Humano. Hojas y Hablas, (10), 54-69.

Chaparro, M., y Urra, M. (2013) Trabajo social en la gestión del talento humano: de lo operativo a lo estratégico. Revista Perspectivas, 24, 145-157.

Chaparro, M., y Urra, M. (2014). Competencias específicas del trabajador social en la gestion del talento humano. Tendencias y retos, 19(2), 40.

Clavijo, J.G. (1989) Identidad cultural del hombre colombiano. Bogotá, Universidad Santo Tomás. Facultad de Sociología, Centro de Investigaciones.

de la Rosa, L. (2007) Impresiones de la comunidad raizal frente a los planteamientos de Peter Wilson. Caribbean Notebooks. Vol 10.

de la Rosa, L. (2012) Les blocs « afro » du carnaval de Salvador de Bahia, entre luttes symboliques et enjeux économiques. Cargo - Revue Internationale d'Anthropologie Culturelle et Sociale, 7 - 19.

de la Rosa, L. (2013) Entre Africanidades y Africanismos: fiestas públicas en Cartagena D Indias, Colombia. Revista Mosaico. Vol 6 (1), pp. 5-16.

de la Rosa, L. (2015) Célébrer la diversité urbaine: groupes carnavalesques" afro" à Salvador de Bahia et à Carthagène des Indes. Tesis doctoral. Disponible en: https://tel.archivesouvertes.fr/tel-01143945

de la Rosa, L. (2017) AS "AÇÕES CARNAVALESCAS": Uma proposta metodológica para abordar a festa a partir de dois exemplos caribenhos. Revista Brasileira do Caribe. Vol 18 (34). Dossier Carnaval e ritmo. Pp. 30-50.

de la Rosa, L. y Moreno, L.M. (2006) Tras la huella de la Candelaria en los litorales colombianos. Memorias, $\mathrm{Vol} 2, \mathrm{~N}^{\circ} 5$.

Ferreras, A., Belda, J.M., Barbera, R., Poveda, R., Urra, M., García, N., y Valero, M. (2010). PDA software aimed at improving workplace adaptation for people with cognitive disabilities. ICCHP'10 Proceedings of the 12th International Conference on Computers Helping People with Special Needs, 13-20. 
Gamba, A. (2006) La Justicia en Equidad como un aporte a la paz sostenible. Artículo disponible en la web: http://www.reddejusticia.org.co

Gamba, A. (2011) Enfoques latinoamericanos en el estudio de los movimientos sociales. Ponencia en Congreso ALAS Recife 2011. Disponible en:

http://www.academia.edu/6337052/Enfoques_latinoamericanos_en_el_estudio_de_los_movi mientos_sociales

Gamba, A. (2013) Democracias restringidas y neoliberalismo en la región andina (1985-2010). Cuadernos Americanos: Nueva Época. Vol 3, no145, pp. 175-194

Gamba, A. (2017) Las acciones colectivas frente a la guerra. El caso del movimiento indígena del Cauca, Colombia. En "¿Estado-Nación o Estsado plural? Pueblos indígenas y el Estado en América Latina (Siglo XXI)", 89-106 . Ciudad de México: UNAM.

Idrobo, J. (2010) El Inteligir En Xavier Zubiri: Aprehensión de la Realidad. Revista de Estudiantes. Universidad Santo Tomás. Vol 1, pp.95-101.

Idrobo, J. (2010) La lógica de una ontología latinoamericana. Revista Electrónica de Investigación de la Universidad Santo Tomás.

Idrobo, J. (2012) Awayu: el tejido de la identidad diversa latinoamericana. Constitución ontológica de una tradición ancestral como posibilidad de interpretación. En Cáceres, I y Quezada, A. (eds) "Identidad... ¿̇latinoamericana? Diálogos de multiplicidades". Pp. 59-70.

Idrobo, J. (2013) A lo profundo de Kusch. Tras una ontología latinoamericana. En "Geopolítica del Hombre Americano".

Idrobo, J. (2013) Caminos de resistencia. Acercamientos al tipo de lucha de los pueblos originarios del Abya Yala. Memorias Periféricas. Vol 5, pp.26-35.

Idrobo, J. (2013) Yachaiwasy: hacia un sentido de ser de la educación latinoamericana desde la liberación. Revista Uturunku Achachi de la Academia Libre y Popular de Humanidades. Vol 2, pp.75-80

Idrobo, J. (2018) Crimen social, justicia transicional y territorio. Apuntes críticos sobre la intervención del "Bronx" en Bogotá. Reflexión y Política, 20 (40),40-49.

Idrobo, J. y Amaya, J. (2018) Reconciliaciones y resistencias. Modelos mentales y aprendizajes colectivos en la construcción de paz territorial en Colombia. Bogotá: Editorial USTA.

J Jaramillo, D Restrepo (2017) Visiones panorámicas sobre la Nueva Sociología Económica. Entrevista al sociólogo Alejandro Portes. Universitas Humanística, 391-399 
Jaime, E. (2013) Jóvenes y justicia restaurativa un proyecto alternativo. Tesis de maestría. Disponible en:

https://repository.javeriana.edu.co/bitstream/handle/10554/15294/JaimeRuizEdwin2013.pdf?s equence $=1$

Jaimes, G. E. (2012). La Guerra Revolucionaria del M-19 (1974-1989). Bogotá , Colombia.

JAP Rueda (2018) Apologías y apocalípticas de la ciudad. El dilema de lecturas sobre la ciudad moderna. Revista Bitácora Urbano Territorial, 20 (1), 85-96.

Leiva E., Andrea (2012) "Apropiación del territorio y espacialidad en el Islote (Caribe colombiano)”. Geopolítica(s). Revista de estudios sobre espacio y poder, vol. 3, núm. 2, 293-328.

Leiva, A. (2012) Cuando Cristo "se metió" a el islote: culto evangélico, atmósfera ritual e interacciones sociales en un territorio insular del Caribe colombiano. Revista Colombiana de Antropología Volumen 48 (2), pp. 39-66

Leiva, A. (2016) " Yo me la paso de isla en isla": formas de habitar e interacciones sociales en el Islote, Caribe colombiano. Tesis doctoral.

Lizarazo Zuluaga Sthefanía (2011) La Bancada de Mujeres del Congreso de Colombia: ¿Un intento de representación? Tesis de Grado. Repositorio Universidad del Valle.

Motoa, J. y F.-X. Tinel. (2009) ¿De vuelta a casa? Reflexiones sobre el retorno de migrantes colombianos y colombianas en España. Diálogos Migrantes. Vol 4, pp. 59-67

Narváez Jaimes, G. E. (2018). Sociología USTA: un compromiso con la vida. Balance, retos y perspectivas. Revista Campos en Ciencias Sociales, 6(2), 119-127. Bogotá

D.C., Colombia: Universidad Santo Tomás. DOI: https://doi.org/10.15332/s2339-

3688.2018.0002.04

Narváez, G. (2012). El populismo armado del movimiento 19 de abril (M-19). Cuadernos de Ciencias Jurídicas y Política Internacional, Vol 5(2), 117-144.

Narváez, G. (2016). Análisis de los Procesos de Paz en Colombia en los inicios de la década de los noventa. Lecciones de El Salvador. Criterios, Vol 7 (2), 43-90.

Narváez, G. (2016). El Consejo de Defensa Suramericano y la creación de una política común de seguridad regional. Criterios, Vol 8 (1), 145-164.

Narváez, G. (2017) Análisis de la ruptura del proceso de negociación entre las FARC-EP y el gobierno colombiano (1999-2002). Tesis doctoral. FLACSO Ecuador. Disponible en:

http://67.192.84.248:8080/bitstream/10469/12756/2/TFLACSO-2017GENJ.pdf 
Ostos, O.L. y Cortés, M.A. (2018) Los campos de acción en la Universidad Santo Tomás. Resultados del estudio 2012 - 2018. Revista Interamericana de Investigación, Educación y Pedagogía, 12 (1) 15-40. DOI: https://doi.org/10.15332/s1657-107X.2019.0001.01

Páez Morales, G., Clavijo Parrado, G., Narváez Jaimes, G. E., Salazar Baena, V., y Urra Canales, M. (2019). Aportes de la Universidad Santo Tomás a la institucionalización de la sociología en Colombia en los años sesenta y setenta. Campos en Ciencias Sociales, 7(1), 227-247. DOI: https://doi.org/10.15332/25006681.4549

Pinzón, J.A. (2012) Modernidad y modernización en la urbanización del tercer mundo. Revista Ciudad Paz-Ando, 5.

Pinzón, J.A. (2012) Reflexiones sobre la urbanización periférica del tercer mundo.

Aproximaciones teóricas para la comprensión del crecimiento de Soacha en la conurbación sur de Bogotá. Tesis. Universidad Nacional de Colombia. Disponible en:

http://www.bdigital.unal.edu.co/11485/1/jorgeandrespinzonrueda.2012.pdf

Sáenz Acosta, H. (2019). El Investigador frente al Espejo. Analizar los Fenómenos Económicos a partir de La Experiencia Propia. International and Multidisciplinary Journal of Social Sciences, 8(3), 191-215. doi: 10.17583/rimcis.2019.4025

Sáenz, H. (2011) Las prácticas del arrendamiento habitacional en 4 barrios populares de Bogotá. Otra economía. Vol 3 (4), pp. 155-173.

Sáenz, H. (2013) La movilidad residencial de los hogares con bajos ingresos y jefatura femenina en el área. Territorios. Vol 28, pp. 207-239.

Sáenz, H. (2015) A reciprocidade como marco institucional dos contratos de locação residencial. Tesis doctoral. Universidade Federal do Rio de Janeiro. Disponible en:

http://objdig.ufrj.br/42/teses/831982.pdf

Sáenz, H. (2016) La subjetivación de las relaciones económicas. Reflexiones para una tipología de mercados informales de arrendamiento residencial. Polis Revista Latinoamericana, 15(45), 235-248.

Sáenz, H. (2018). El arrendamiento residencial en los barrios populares: las redes de relaciones y la generación de compromisos. Territorios, (38), 95-117. Doi:

http://dx.doi.org/10.12804/revistas.urosario.edu.co/territorios/a.5999

Sáenz,H. (2010) El arrendamiento residencial en los barrios populares: las redes de relaciones y la generación de compromisos. Territorios. Vol 38, pp. 95-117.

Sáenz,H. (2010) La ilusión de la participación comunitaria. Lucha y negociación en los barrios populares de Bogotá 1992-2003. Territorios. Vol 23, pp. 161-165. 
Sáenz,H. (2018) El arrendamiento residencial en los barrios populares: las redes de relaciones y la generación de compromisos. Territorios. Vol 38, pp. 95-117.

SALAZAR BAENA, VERÓNICA, \& HERRERO SÁNCHEZ, MANUEL. (2018). Repúblicas Y republicanismo en la Europa moderna (siglos XVI-XVIII). Fronteras de la Historia, 23(1), 240-250. https://dx.doi.org/10.22380/20274688.323

Salazar, V. (2012) Las ceremonias reales en el virreinato de la Nueva Granada. Tesis de maestría. Ponencia. Disponible en: http://hdl.handle.net/10261/73287

Salazar, V. (2014) Fastos monárquicos en el Nuevo Reino de Granada. La imagen del rey y los intereses locales. Siglos XVII-XVIII. Tesis Doctoral. Disponible en: diposit.ub.edu/dspace/bitstream/2445/53353/1/VSB_TESIS.pdf

Salazar, V. (2017) El cuerpo del rey: poder y legitimación en la monarquía hispánica. Fronteras de la Historia, 22 (2) pp.140-168

Tinel, F.X. (2008) Las voces del silencio. Resistencia indígena en Chimborazo en tiempos de León Febres Cordero, 1984-1988, Quito, Flacso-Abya-Yala.

Tinel, F.X. (2015) Desarrollo e hiperconsumo: la producción de lo efímero. Campos de Ciencias Sociales. Vol 3 (1) pp.79-96.

Torres C.A., Vargas J.E. (2009) Vivienda para población desplazada en Colombia: recomendaciones para la política pública y exigibilidad del derecho. Revista INVI. Vol 24 (66), pp.17-86.

Torres Carrillo, A. y Torres Ruiz, A.C. (2015) Acción colectiva, gestión territorial y gobernanza democrática en Bogotá. Bogotá: Universidad Piloto de Colombia.

Torres Carrillo, A. y Torres Ruiz, A.C. (2015) Actores prácticas y sentidos de la participación local en Bogotá. Bogotá: Universidad Piloto de Colombia.

Torres Ruiz, A.C. (2007) Conflicto y justicia, una perspectiva anamnético-narrativa. Theologica Xaveriana. Vol 57 (163)

Torres Ruiz, A.C. (2017) Infraestructuras urbanas sociotécnicas. Vender o no vender la ETBEmpresa de Telecomunicaciones de Bogotá. Revista Brasileira de Gestão Urbana. Vol 4 (2), pp.263-297.

Torres Tovar, C. A., Amaya Medina, N., Rincón García, J. J., \& Vargas Moreno, J. E. (2013). Hacer del Mejoramiento Barrial y Urbano una política pública estratégica para la superación de la pobreza. Bitacora 23. Pp. 105-114 
Torres Tovar, C.; Rincón García, J. y Vargas Moreno, J. (2009). Pobreza urbana y mejoramiento integral de barrios: hábitat y vivienda. Bogotá, Colombia: Universidad Nacional de Colombia. Recuperado de http://www.facartes.unal.edu.co/otros/libros_habitat/ pobreza_urbana.pdf

Urra Canales, M., Acosta Oidor, C., Salazar Baena, V., Jaime Ruiz, E. (2018). Bullying. Description of the Roles of Victim, Bully, Peer Group, School, Family and Society. International Journal of Sociology of Education, 7(3), 278-299. doi: 10.17583/rise.2018.3547

Urra, M. (2010) El papel de la economía social en los regímenes del bienestar. Revista Miscelánea Comillas, vol. 68, n. 133, pp. 791-816.

Urra, M. (2012) Especificidad del trabajo social en la RSE de empresas colombianas. Tendencias \& Retos. Vol. 17, nำ2, pp. 79-88

Urra, M. (2012) La gestión del talento humano, un reto para el profesional de Trabajo Social. Hojas y Hablas, (9), 102-111.

Urra, M. (2012) Trabajo Social en el ámbito empresarial, proceso histórico, definiciones y tendencias. Hojas y Hablas, (9), 91-96.

Urra, M. (2017) Bullying Acoso Escolar. Retrieved from osf.io/preprints/socarxiv/fxsy3

Urra, M. (2018) Estado, mercado, academia... y comunidad. Una cuádruple hélice para el desarrollo integral y la innovación. Tesis. Universidad Pontificia de Comillas. Disponible en: http://hdl.handle.net/11531/26826

Urra, M. (2018) Paradigma de desarrollo social y humano. Orígenes y pioneros. Retrieved from osf.io/preprints/socarxiv/ntfer DOI: 10.17605/OSF.IO/NTFER

Urra, M. et ál. (2019). La revista Cuadernos de Sociología de la Universidad Santo Tomás (Bogotá, Colombia, 1977-2011). Revista Colombiana de Sociología, 42(2),

DOI: $10.15446 /$ rcs.v42n2.77191

Varón, D.C (2014) La vida es mejor con audífonos Análisis de las prácticas de escucha ligadas al uso de la tecnología en el transporte público de Bogotá. Tesis de Maestría. Disponible en: https://repository.javeriana.edu.co/bitstream/handle/10554/13466/VaronCastiblancoDianaCar olina2014.pdf?sequence=1 
